\title{
Ionization of water molecules by proton impact: Two nonperturbative studies of the electron-emission spectra
}

\author{
L. F. Errea, Clara Illescas, L. Méndez, and I. Rabadán \\ Laboratorio Asociado al CIEMAT de Física Atómica y Molecular en Plasmas de Fusión, Departamento de Química, módulo 13, \\ Universidad Autónoma de Madrid, Cantoblanco E-28049 Madrid, Spain
}

(Received 26 September 2012; published 18 March 2013)

\begin{abstract}
Two nonperturbative methods are applied to obtain total and singly differential (in the electron energy) cross sections of electron emission in proton collisions with $\mathrm{H}_{2} \mathrm{O}$ at impact energies in the range $10 \mathrm{keV} \leqslant E_{\mathrm{p}} \leqslant$ $5 \mathrm{MeV}$. Both methods, one classical and one semiclassical, combine an independent particle treatment with a multicenter model potential description of the target. The excellent agreement obtained with experimental data supports the usefulness of the approximations involved and encourages the study of more complex systems.
\end{abstract}

DOI: 10.1103/PhysRevA.87.032709

PACS number(s): $34.50 . \mathrm{Gb}, 34.70 .+\mathrm{e}$

\section{INTRODUCTION}

The ionization of water molecules by colliding ions is a fundamental process in describing the interaction of ion beams with biological environments, which is particularly important in cancer hadron therapy (see Ref. [1]). Since water is the main constituent of the cell, the biological damage is caused, to a large extent, by secondary particles (electrons, ions, and radicals) formed by ion collisions with $\mathrm{H}_{2} \mathrm{O}$ (see [2] and references therein); accurate cross sections for these atomic processes are therefore required to simulate the passage of ions through biological environments. These applications have motivated several perturbative calculations [3-8] of total and differential ionization cross sections in ion $+\mathrm{H}_{2} \mathrm{O}$ collisions, which are in general valid for relatively high collision energies.

In order to extend the existent theoretical data down to collision energies above $10 \mathrm{keV}$, and to simultaneously evaluate total cross sections for electron capture and ionization reactions, we have developed a classical trajectory Monte Carlo (CTMC) treatment [9-11] that has been successfully applied to calculate total cross sections for $\mathrm{H}^{+}, \mathrm{He}^{2+}$, and $\mathrm{C}^{6+}$ collisions with $\mathrm{H}_{2} \mathrm{O}$. More recently, Murakami et al. [12,13] have studied $\mathrm{H}^{+}+\mathrm{H}_{2} \mathrm{O}$ collisions by employing a semiclassical treatment with a collision wave function expanded in a two-center basis set, augmented by a set of target pseudostates. These pseudostates are constructed by applying the basis generator method (BGM), where they are obtained by repeated application of the (regularized) electron-projectile interaction potential on the target orbitals (see [14] and references therein).

Previous calculations of Refs. $[9,10,12,13]$ provided total cross sections for both single ionization (SI) and electron production (EP) processes. EP (also called net ionization) includes all reactions where electrons are emitted, mainly SI,

$$
\mathrm{A}^{q+}+\mathrm{H}_{2} \mathrm{O} \rightarrow \mathrm{A}^{q+}+\mathrm{H}_{2} \mathrm{O}^{+}+e^{-},
$$

double ionization (DI),

$$
\mathrm{A}^{q+}+\mathrm{H}_{2} \mathrm{O} \rightarrow \mathrm{A}^{q+}+\mathrm{H}_{2} \mathrm{O}^{2+}+2 e^{-},
$$

and transfer ionization (TI),

$$
\mathrm{A}^{q+}+\mathrm{H}_{2} \mathrm{O} \rightarrow \mathrm{A}^{(q-1)+}+\mathrm{H}_{2} \mathrm{O}^{2+}+e^{-} .
$$

Both Murakami et al. [12] and Illescas et al. [10] report total EP cross sections in generally good agreement with the experiments [15-18], although the maximum of the cross section in the BGM calculations appears at lower impact energy than those of different experiments and theories (continuum distorted wave eikonal initial state [3] and CTMC [10]); the BGM calculation also overestimates the experimental cross section at collision energies below $50 \mathrm{keV}$. However, the magnitudes of the contributions of SI and multiple electron processes to EP in Refs. [10,12] clearly disagree. In order to further discuss these results, one must take into account that, as pointed out in [12], it is difficult to compare theoretical SI cross sections with the experiments that measure the formation of different molecular cations produced in the collision. Nonetheless, the experiments that report EP total cross sections are directly related to the calculations, and the comparison can provide useful information on the accuracy of the theoretical methods.

Although previous applications of the CTMC and semiclassical methods have focused on obtaining total cross sections, they can also be applied to calculate differential cross sections. Our goal is to calculate single-differential cross sections (SDCS) and to compare them with available experiments $[15,16,19]$; this is a stringent test of the methods employed here and, at the same time, provides necessary data for the codes simulating ion tracks in biological media [20-24]. It is important to note that, until now, only the calculations based on the first Born approximation of Refs. [5,8] (for $\mathrm{H}^{+}+\mathrm{H}_{2} \mathrm{O}$ ), [6] (for $\mathrm{He}^{2+}+\mathrm{H}_{2} \mathrm{O}$ ) and [7] (for $\mathrm{C}^{6+}+\mathrm{H}_{2} \mathrm{O}$ ) have evaluated SDCS for $E_{\mathrm{p}}>100 \mathrm{keV} / \mathrm{u}$, and that while SDCS in ionization of water molecules by protons were measured more than 25 years ago, only recently some calculations have been carried out.

\section{THEORETICAL METHODS}

\section{A. CTMC treatment}

The main assumptions of our CTMC treatment (see Ref. [10] for details), are

1. The water nuclei are fixed in their equilibrium positions and the projectile follows rectilinear trajectories: $\boldsymbol{R}=\boldsymbol{b}+\boldsymbol{v} t$.

2. The independent electron approximation is applied, and the active electron initially moves in the average potential created by the nuclei and the other electrons of the target molecule; a three-center model potential in our calculation. 
3. Each target valence molecular orbital (MO) $k$ is described by means of a microcanonical classical distribution function $\rho_{k}(\boldsymbol{r}, \boldsymbol{p})$, build out of $N\left(\approx 10^{5}\right)$ trajectories with the same energy $E_{k}$.

4. The ionization probabilities of the many-electron system are obtained from the one-electron ionization probabilities using the independent event model (IEVM) (see Ref [25]). In practice (see [10]), the two electrons occupying the same MO can be simultaneously ejected, while the probability of ejecting two electrons from different shells is neglected. In general, this approximation can be applied when the electron ejection, either by capture or ionization, from different MOs take place at well separated points of the nuclear trajectory or for different molecular orientations with respect to the nuclear trajectory. Neglecting the ionization probabilities of core electrons, the SI probability is given by

$$
P^{\mathrm{SI}}(\boldsymbol{b}, \boldsymbol{v})=2 \sum_{k=1}^{4} p_{k}(\boldsymbol{b}, \boldsymbol{v}) p_{k}^{\mathrm{el}}(\boldsymbol{b}, \boldsymbol{v}),
$$

where $p_{k}$ is the ionization probability of the electron in the $k$ th $\mathrm{MO}$ and $p_{k}^{\mathrm{el}}$ is the probability that the electron is neither captured nor ionized. Within this approximation, the EP probability includes one- and two-electron transitions (SI, DI, and TI) and has the form

$$
\begin{gathered}
\tilde{P}^{\mathrm{EP}}(\varepsilon, \boldsymbol{b}, \boldsymbol{v})=2 \sum_{k=1}^{4} \tilde{p}_{k}(\varepsilon, \boldsymbol{b}, \boldsymbol{v}), \\
P^{\mathrm{EP}}(\boldsymbol{b}, \boldsymbol{v})=2 \sum_{k=1}^{4} p_{k}(\boldsymbol{b}, \boldsymbol{v}) .
\end{gathered}
$$

where $\tilde{p}_{k}$ and $\tilde{P}^{\mathrm{EP}}$ are ionized-electron-energy $(\varepsilon)$ dependent probabilities.

In the CTMC treatment, the one-electron probabilities $\tilde{p}_{k}$ and $p_{k}$ are evaluated from the number of electron trajectories, $n_{k}\left(\varepsilon_{i}\right)$, whose energy ends up in the bin with energy $\varepsilon_{i}$ and width $\Delta \varepsilon_{i}$, and the total number of ionized trajectories, $m_{k}$ :

$$
\begin{gathered}
\tilde{p}_{k}\left(\varepsilon_{i}, \boldsymbol{b}, \boldsymbol{v}\right)=\frac{n_{k}\left(\varepsilon_{i}, \boldsymbol{b}, \boldsymbol{v}\right)}{N}, \\
p_{k}(\boldsymbol{b}, \boldsymbol{v})=\frac{m_{k}(\boldsymbol{b}, \boldsymbol{v})}{N} .
\end{gathered}
$$

Here, as $\varepsilon$ may span several orders of magnitude, we employ a binning technique in which the bin width $\Delta \varepsilon$ is proportional to $\varepsilon$. Specifically, if we collect trajectories with final energy between $\varepsilon_{\min }$ and $\varepsilon_{\max }$ in $n$ bins, the energy of bin $i(=1, \ldots, n)$ is $\varepsilon_{i}=\varepsilon_{\min } \gamma^{i / n-1 / 2 n}$ with $\gamma=\varepsilon_{\max } / \varepsilon_{\min }$, the $i$ bin goes from $\varepsilon_{i} \gamma^{-1 / 2 n}$ to $\varepsilon_{i} \gamma^{1 / 2 n}$, and, consequently, $\Delta \varepsilon_{i}=\varepsilon_{i}\left(\gamma^{1 / 2 n}-\gamma^{-1 / 2 n}\right)$.

\section{B. Semiclassical treatment}

We have also carried out a semiclassical treatment as in $[11,26]$. This method also applies previous assumptions 1,2 , and 4 and uses one-electron scattering wave functions expanded in terms of asymptotic-frozen molecular orbitals (AFMO) $\left\{\chi_{i}(\boldsymbol{r})\right\}$ of $\mathrm{H}_{3} \mathrm{O}^{+}$:

$$
\Psi_{k}(\boldsymbol{r}, t, \boldsymbol{b}, \boldsymbol{v})=\sum_{i=1} a_{k i}(t, \boldsymbol{b}, \boldsymbol{v}) \chi_{i}(\boldsymbol{r}) e^{-i \int^{t} \lambda_{i i} d t^{\prime}},
$$

where $\boldsymbol{\lambda}=\mathbf{S}^{-\mathbf{1}} \mathbf{H}$, and $\mathbf{S}$ and $\mathbf{H}$ are the overlap and Hamiltonian matrices in the AFMO basis. This method is an extension to ion-molecule collisions of the atomic expansion (see, e.g., [27]), widely used in ion-atom collisions. As in the CTMC treatment, the index $k$ indicates the initially occupied MO. Our molecular basis is relatively large and consists of 215 AFMOs constructed using a basis set of Gaussian type orbitals (GTOs) centered on the nuclei. 140 AFMOs of the basis set have asymptotical positive energy and furnish a discretization of the electronic continuum. The GTO basis set employed here is the augmented correlation-consistent, polarization valence triple zeta (aug-cc-pVTZ) of [28,29] for oxygen and hydrogen atoms of the water molecule, enlarged with $(4 s 4 p 4 d 3 f)$ and $(2 s 1 p)$ sets of diffuse functions, respectively. For the hydrogen projectile, we have constructed a new basis set consisting of $[6 s, 4 p, 2 d]$ contracted GTOs. This basis and the exponents of the set of extra diffuse functions employed can be found in the complementary material [30].

We have not included translation factors in (9), because, as explained in Ref. [31], they do not significantly improve the quality of the basis set when ionization is the dominant process. A more correct description of the ionizing wave function would need to reproduce the free expansion phase related to the quasifree expansion of the electron cloud seen in classical treatments [32]. However, the lack of translation factors in this expansion leads to a noticeable overestimation of the electron capture probabilities, and therefore it is not appropriate to evaluate electron capture cross sections. The one-electron probability of finding the electron in the energy bin $\varepsilon_{i}$, after starting in the orbital $k$, is

$$
\tilde{p}_{k}\left(\varepsilon_{i}, \boldsymbol{b}, \boldsymbol{v}\right)=\sum_{j}\left|a_{k j}(t \rightarrow \infty, \boldsymbol{b}, \boldsymbol{v})\right|^{2},
$$

with $j$ running over AFMOs with asymptotic values of $H_{j j}$ within the energy bin $i$, while

$$
p_{k}(\boldsymbol{b}, \boldsymbol{v})=\sum_{j}\left|a_{k j}(t \rightarrow \infty, \boldsymbol{b}, \boldsymbol{v})\right|^{2},
$$

with $j$ running over all AFMOs with $H_{j j}>0$.

In both CTMC and semiclassical calculations, we have evaluated SI and EP probabilities by using Eqs. (4)-(6), and in both treatments we have evaluated orientation averaged SDCS and total cross sections by assuming that the molecule is fixed in space while we average with respect to the orientation of the ion trajectory, defined by $(\hat{\boldsymbol{b}}, \hat{\boldsymbol{v}})$, with $\hat{\boldsymbol{b}} \perp \hat{\boldsymbol{v}}$. Explicitly,

$$
\begin{gathered}
\frac{d \sigma^{\mathrm{EP}}}{d \varepsilon}\left(\varepsilon_{i}, v\right)=\frac{1}{4 \pi \Delta \varepsilon_{i}} \int d \Omega \int \tilde{P}^{\mathrm{EP}}\left(\varepsilon_{i}, \boldsymbol{b}, v, \Omega\right) d \boldsymbol{b}, \\
\sigma^{\mathrm{EP}, \mathrm{SI}}(v)=\frac{1}{4 \pi} \int d \Omega \int P^{\mathrm{EP}, \mathrm{SI}}(\boldsymbol{b}, v, \Omega) d \boldsymbol{b},
\end{gathered}
$$

where $\Omega$ is the solid angle that defines the direction of $v$ with respect to the molecular target. The integration has been performed numerically by applying the method of Refs. [33,34], where the orientation average is calculated by considering 12 projectile trajectory orientations (actually 10 different ones when taking into account the molecular 
symmetry [11]):

$$
\begin{aligned}
\frac{d \sigma^{\mathrm{EP}}}{d \varepsilon}\left(\varepsilon_{i}, v\right) & =\frac{1}{12} \sum_{m=1}^{12}\left(\frac{d \sigma^{\mathrm{EP}}}{d \varepsilon}\right)_{m}, \\
\sigma^{\mathrm{EP}, \mathrm{SI}}(v) & =\frac{1}{12} \sum_{m=1}^{12} \sigma_{m}^{\mathrm{EP}, \mathrm{SI}},
\end{aligned}
$$

with

$$
\begin{aligned}
\left(\frac{d \sigma^{\mathrm{EP}}}{d \varepsilon}\right)_{m} & =2 \pi \int_{0}^{\infty} b \tilde{P}_{m}^{\mathrm{EP}}\left(\varepsilon_{i}, b, v\right) d b, \\
\sigma_{m}^{\mathrm{EP}, \mathrm{SI}} & =2 \pi \int_{0}^{\infty} b P_{m}^{\mathrm{EP}, \mathrm{SI}}(b, v) d b,
\end{aligned}
$$

and the probabilities $\tilde{P}_{m}^{\mathrm{EP}}$ and $P_{m}^{\mathrm{EP}, \mathrm{SI}}$ are evaluated for a given trajectory orientation.

\section{RESULTS}

\section{A. Classical and semiclassical initial momentum distributions}

As it is well known, the accuracy of the CTMC method strongly depends on that of the initial position and momentum distributions. In Ref. [10], we compared the classical spatial distributions with quantal probability distributions of the water MOs and we found a very good global description of the quantal density. It must be noted that the well known inability of the microcanonical distribution to describe the tail of the spatial quantal distribution is relatively less important for $n=$ 2 than for $n=1$ orbitals (see, e.g., [35]), and the valence MOs of water are essentially $n=2$ orbitals of the oxygen atom; this drawback is, to some extent, compensated by the use of a threecenter potential. Although in one-electron atoms the classical (microcanonical) momentum distribution function is exact, for the $\mathrm{H}_{2} \mathrm{O}$ system the distribution is defined numerically and an analogous property probably does not hold. Accordingly, we have compared the initial classical momentum distributions

$$
\rho_{k}^{\mathrm{C}}(\boldsymbol{p})=\int d \boldsymbol{r} \rho_{k}(\boldsymbol{r}, \boldsymbol{p})
$$

with the corresponding quantal ones

$$
\rho_{k}^{\mathrm{Q}}(\boldsymbol{p})=\left|\int \exp (-i \boldsymbol{p} \cdot \boldsymbol{r}) \chi_{k}(\boldsymbol{r}) d \boldsymbol{r}\right|^{2} .
$$

The comparison is illustrated in Fig. 1 for the two highest occupied MOs of $\mathrm{H}_{2} \mathrm{O}\left(1 b_{1}\right.$ and $\left.3 a_{1}\right)$, from which the electrons are emitted with the largest probabilities [10]. We observe in this figure a very good agreement for the components $p_{x}$ and $p_{z}$ of the $1 b_{1}$ orbital, and for $p_{x}$ and $p_{y}$ of the $3 a_{1}$ one. The situation is similar to that found for the spatial distributions in [10]: the main limitation of the initial classical distribution is the impossibility of reproducing the nodes of the quantal one, but this limitation is smeared out when one calculates orientation averaged cross sections and includes transitions from all valence MOs.

\section{B. Total SI and EP cross sections}

SI total cross sections for both CTMC and AFMO treatments are shown in Fig. 2(a), where they are compared with BGM calculations of Ref. [12] and the experimental results.
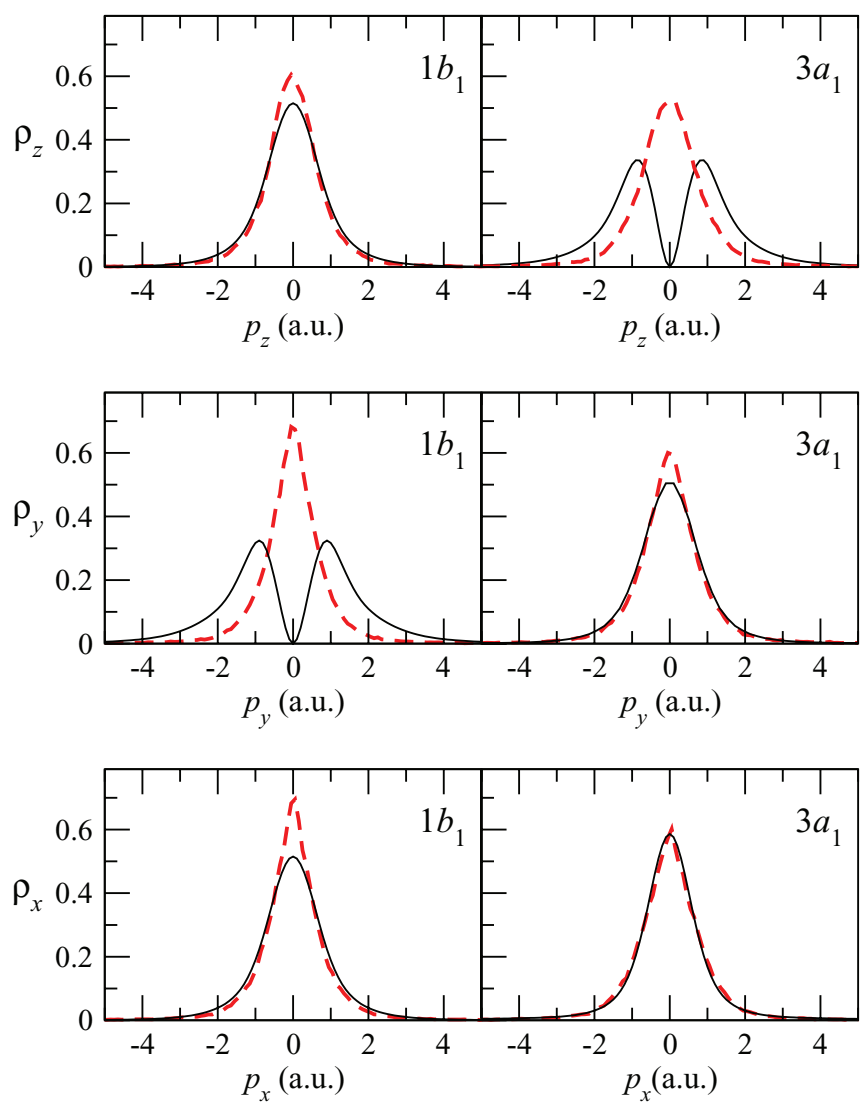

FIG. 1. (Color online) Cartesian components of the classical [dashed lines, Eq. (16)] and quantal [solid lines, Eq. (17)] electronic momentum densities of the two highest occupied molecular orbitals of the water molecule $\left(1 b_{1}\right.$ and $\left.3 a_{1}\right)$.

As already mentioned, the $\mathrm{SI}$ of $\mathrm{H}_{2} \mathrm{O}$ yields $\mathrm{H}_{2} \mathrm{O}^{+}$that, in a post-collisional process, can fragment into singly charged ions: $\mathrm{H}^{+}, \mathrm{OH}^{+}$, and $\mathrm{O}^{+}[36]$. Within this two-step mechanism, the branching ratios for formation of these cations must be similar to those measured in photoionization experiments [37]. The total SI cross sections from Ref. [12] agree with those measured in $[17,18,38,39]$ for formation of $\mathrm{H}_{2} \mathrm{O}^{+}$. Since the fragmentation of the water cation is not negligible, this comparison indicates, as also pointed out by the authors, that the SI total cross section of Ref. [12] is a lower bound of the real one. On the other hand, the SI total cross sections of Ref. [10] (CTMC) agree with the sum of the cross sections for formation of all singly charged species, which points to them being upper bound values, given that $\mathrm{H}^{+}, \mathrm{OH}^{+}$, and $\mathrm{O}^{+}$ could also be produced by fragmentation of $\mathrm{H}_{2} \mathrm{O}^{2+}$, formed by a two-electron process. It can be noted that the new AFMO results reasonably agree with the CTMC ones, which indicates that the classical approximation is not responsible for the disagreement with Ref. [12].

In order to further study these results, we have plotted in Fig. 2(a) total SI cross sections evaluated by using the independent particle interpretation (IPM), in which all the valence electrons are equivalent:

$$
P^{\mathrm{SI}}(\boldsymbol{b}, \boldsymbol{v})=2 \sum_{k=1}^{4} p_{k}(\boldsymbol{b}, \boldsymbol{v}) p_{k}^{\mathrm{el}}(\boldsymbol{b}, \boldsymbol{v}) \prod_{j=1, j \neq k}^{4}\left[p_{j}^{\mathrm{el}}(\boldsymbol{b}, \boldsymbol{v})\right]^{2} .
$$



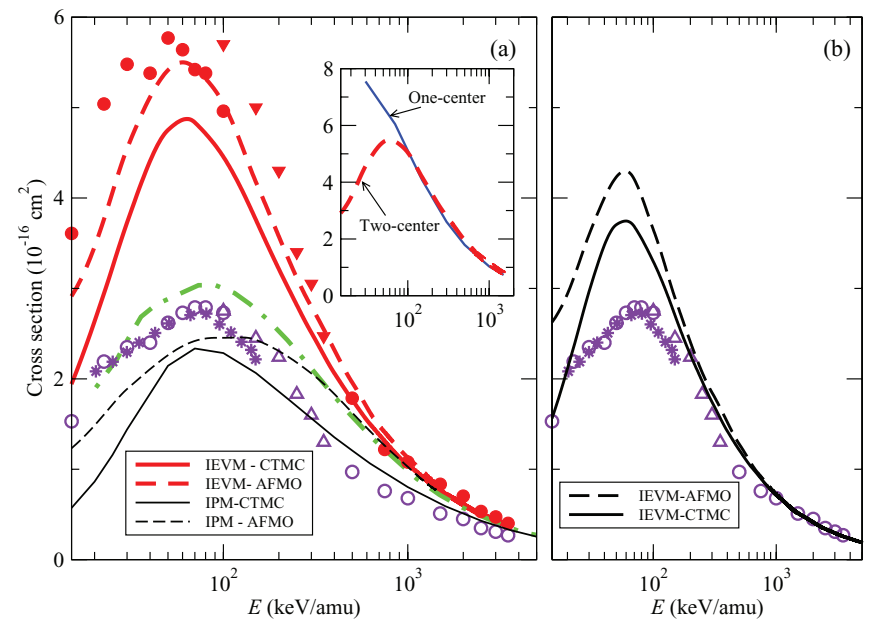

FIG. 2. (Color online) (a) Total cross sections for single ionization, $\sigma^{\mathrm{SI}}$, in proton collisions with water molecules as functions of the collision energy. Present CTMC and semiclassical calculations are compared with the theoretical work of Ref. [12] (dashed-dotted line) and measurements: ॰, • [18]; $\triangle, \boldsymbol{\nabla}$ [38]; * [17]. Full symbols correspond to the sum of cross sections for formation of $\mathrm{H}_{2} \mathrm{O}^{+}, \mathrm{OH}^{+}$, $\mathrm{O}^{+}$, and $\mathrm{H}^{+}$; empty symbols and stars are the cross sections for formation of $\mathrm{H}_{2} \mathrm{O}^{+}$. In the inset we compare the semiclassical results with one- and two-center basis sets (see text). (b) Total cross sections for production of $\mathrm{H}_{2} \mathrm{O}^{+}$in single ionization of $\mathrm{H}_{2} \mathrm{O}$. The lines are the CTMC and AFMO results with the IEVM interpretation and using Eq. (19).

The use of expression (18) leads to a noticeable decrease of the cross sections with respect to those evaluated using (4), a consequence of allowing the simultaneous ionization of electrons from different MOs. It can also be noticed that the IPM cross sections reasonably agree with those of Ref. [12] that employs a similar interpretation of the one-electron probabilities, although this work explicitly takes into account the influence of the Pauli principle, not considered in our AFMO calculation. The comparison with the experiments seems to indicate that the IPM interpretation underestimates the SI cross section, given that the three calculations that employ this interpretation yield cross sections close to the experimental one, $\sigma^{\mathrm{SI}}\left(\mathrm{H}_{2} \mathrm{O}^{+}\right)$, for formation of $\mathrm{H}_{2} \mathrm{O}^{+}$, while other singly charged fragments are produced in this reaction. On the other hand, the total IEVM-CTMC SI cross section lies below the experimental cross sections for formation of all singly charged species, which can be explained with a relatively small contribution of singly charged cations originating in multiple ionization reactions, as suggested in Ref. [12].

A complementary comparison is shown in Fig. 2(b), where we have plotted the calculated cross section $\sigma^{\mathrm{SI}}\left(\mathrm{H}_{2} \mathrm{O}^{+}\right)$ obtained by combining the IEVM cross sections for ionization of electrons from individual MOs with the experimental branching ratios of Tan et al. [37]:

$$
\sigma^{\mathrm{SI}}\left(\mathrm{H}_{2} \mathrm{O}^{+}\right)=\sigma\left(1 b_{1}\right)+\sigma\left(3 a_{1}\right)+0.080 \sigma\left(1 b_{2}\right) .
$$

These cross sections show a general good agreement with the experimental data, particularly for $E \gtrsim 100 \mathrm{keV}$, where the relatively small ionization probabilities support the use of Eq. (4) and fragmentation during the collision is unlikely. The discrepancy between CTMC and AFMO in Figs. 2(a) and 2(b) at low energies is probably due to an overestimation of the cross section in the semiclassical calculation because the ionizing flux is not completely separated from that leading to electron capture. The interlocking between capture and ionization mechanisms happens because the traveling orbitals of the projectile are partially described by the target pseudostates. A useful test on the relevance of this effect is shown in the inset of Fig. 2(a), where we present the results of one-center calculation with a basis set allocated only on the molecular target. It is clear that the ensuing SI cross section increases with respect to the corresponding two-center calculation at energies below the maximum of the SI cross section; however, the agreement between both results at $E \gtrsim 60 \mathrm{keV}$ indicates that the contamination by the capture process of the SI cross section is negligible at these energies. Furthermore, the one-center calculation does not require us to include electron translation factors, and this illustration shows that neglecting the electron translation factors does not limit the validity of expansion (9) at high energies to calculate the ionization cross section.

Our implementation of the IEVM assumes that the probabilities of three or more electron ionization processes vanish and, therefore, the EP probabilities are

$$
P^{\mathrm{EP}}=P^{\mathrm{SI}}+P^{\mathrm{TI}}+2 P^{\mathrm{DI}}
$$

which yieds Eq. (6). In the IPM treatment multiple ionization occur and the EP probability is then given, in our case, by

$$
P^{\mathrm{EP}}=\sum_{q=1}^{8} q P^{q},
$$

where $P^{q}$ is the probability for releasing $q$ electrons to the continuum. It can be proved (see also [40]) that (21) also leads to Eq. (6) and, consequently, IEVM and IPM interpretations differ in the probabilities of specific contributions, but not in the net ionization probability. Besides, unlike the situation

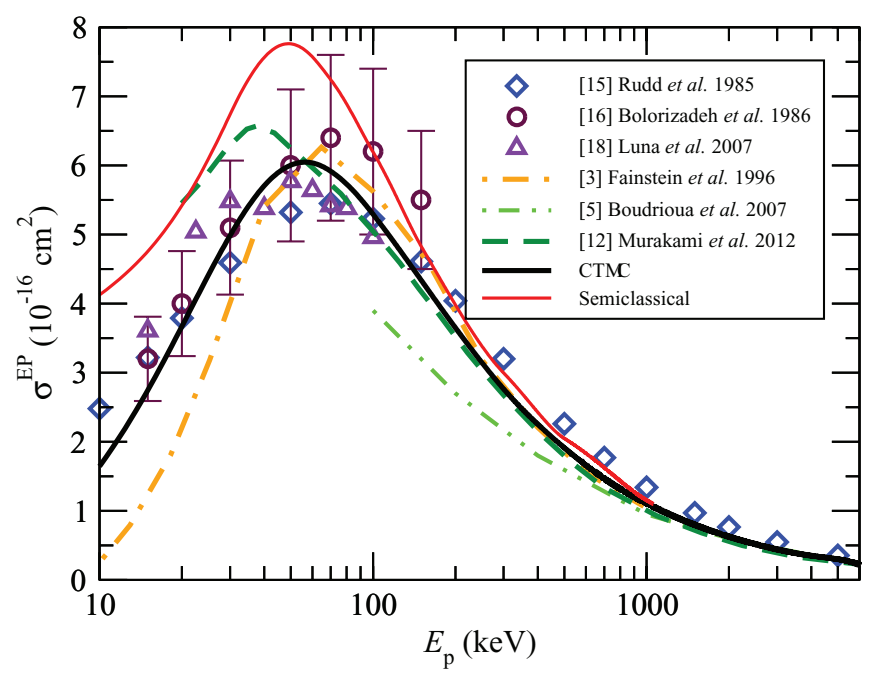

FIG. 3. (Color online) Total cross sections for electron production, $\sigma^{\mathrm{EP}}$, in proton collisions with water molecules as functions of the collision energy. Present CTMC and semiclassical calculations are compared with previous theoretical works $[3,5,12]$ and measurements $[15,16,18]$, as indicated in the figure. 


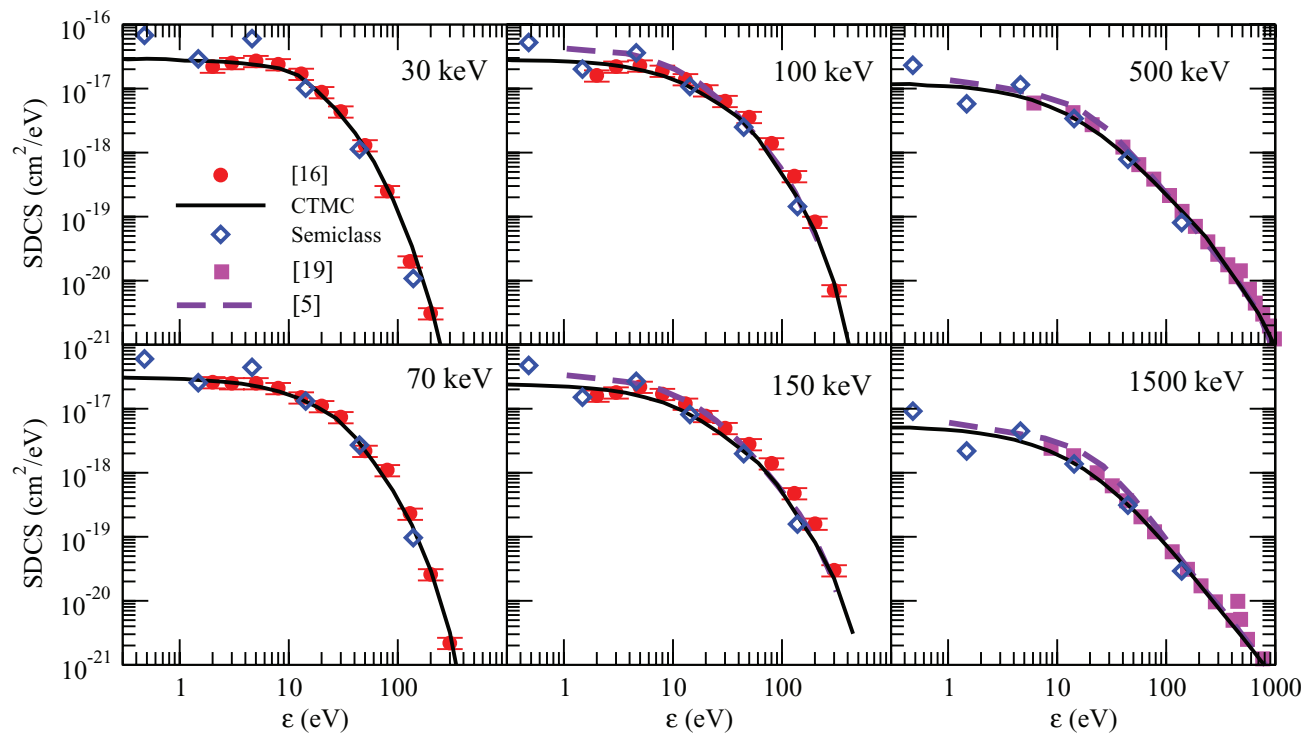

FIG. 4. (Color online) SDCS for electron production, $\sigma^{\mathrm{EP}}$, in proton collisions with $\mathrm{H}_{2} \mathrm{O}$ as functions of the energy of the emitted electron. The projectile energy $E_{\mathrm{p}}$ is indicated in each panel. Included in the figure are the present CTMC and semiclassical results, the experimental data of Refs. [16,19], and the perturbative calculation of Ref. [5], as indicated in the figure.

found for SI, experimental EP cross sections are independent of the post-collisional fragmentation of molecular cations, which allows a direct comparison with calculations.

We compare in Fig. 3 the total EP cross section, $\sigma^{\mathrm{EP}}$, evaluated using our classical and semiclassical approaches, with the experimental $[15,16,18]$ and other theoretical $[3,5,12]$ works. In this illustration, the data of Ref. [18] correspond to the sum of the cross section for SI and TI. Perturbative calculations are appropriate at high impact energies with the exception of that of Ref. [3], which is able to reproduce the maximum of $\sigma^{\mathrm{EP}}$, although it underestimates this cross section at $E_{\mathrm{p}} \leqslant 60 \mathrm{keV}$. The results of Ref. [4], not included in the figure, are indistinguishable from our CTMC ones for $E_{\mathrm{p}} \geqslant$ $300 \mathrm{keV}$. The semiclassical treatment leads to cross sections that show general good agreement with the experiments and with the CTMC results for $E_{\mathrm{p}}>100 \mathrm{keV}$, while at lower energies the semiclassical calculation overestimates the cross section, reproducing the behavior already found in the SI cross section. One can note a similar, although less marked, behavior of the BGM calculation. The general good agreement between the semiclassical and CTMC calculations supports both the validity of the classical approximation and the convergence of the basis set in the semiclassical calculation to globally describe the electronic continuum. Moreover, the good agreement between both calculations and the experimental data for $E_{\mathrm{p}}>100 \mathrm{keV}$ confirms the accuracy of the independent electron approximation, as employed in Eqs. (5) and (6), which is applied in both of them and also in the calculations of Refs. $[3,5,12,41]$.

\section{Electron emission spectra}

The electron emission spectra obtained with both CTMC and semiclassical treatments are shown in Fig. 4. In the semiclassical calculation, the accuracy of the result is determined by a uniform density of states that is not easy to obtain from a set of atomic orbitals, which must also accurately describe the bound states of the four-center collision system. In this respect, the oscillation of the semiclassical points at low $\varepsilon$ is a consequence of the relatively small number of levels in the corresponding energy bin, and we have checked that its shape changes when either the bins boundaries or the basis are modified. We find that for $\varepsilon \geqslant 10 \mathrm{eV}$ the semiclassical results show very good agreement with the CTMC calculations at all impact energies considered, while they oscillate around the CTMC ones for $\varepsilon<10 \mathrm{eV}$. Furthermore, for $E_{\mathrm{p}}<100 \mathrm{keV}$, the overestimation of the total EP cross section by the semiclassical calculation is also observed in the SDCS at $\varepsilon<10 \mathrm{eV}$. The semiclassical calculation provides, however, a valuable check of the magnitude and shape of the classical SDCS and the experimental values. In particular, the comparisons presented in Fig. 4 support the application of the CTMC method to evaluate $\operatorname{SDCS}$ at low $\varepsilon$, which are specially relevant since low-energy electrons $(\varepsilon \approx 10 \mathrm{eV})$ are responsible for DNA strand break [42].

It can also be noted in Fig. 4 that the experimental SDCS of Bolorizadeh and Rudd [16] exhibit a maximum at $\varepsilon \approx 5 \mathrm{eV}$, which is not found in the CTMC calculations, and that is not observed in the published experimental data at $E_{\mathrm{p}}>0.3 \mathrm{MeV}$ of Toburen and Wilson [19]. In this respect, the experimental data plotted in Fig. 4 were provided by Prof. Toburen [43] who also pointed out that these measurements have a limited accuracy at $\varepsilon<10 \mathrm{eV}$. The CTMC cross sections show general good agreement with the first Born ones of [5] with the exception of the low-energy region, $\varepsilon<10 \mathrm{eV}$, where the perturbative calculation yields cross sections systematically higher than ours. This comparison seems to indicate that the low- $\varepsilon$ range was not completely included in the integration over $\varepsilon$ of Ref. [5], which leads to a total cross section lower than ours and the experimental ones (see Fig. 3). The fact that the CTMC SDCS for ionization processes of water molecules by protons presented in this work show very good agreement 
with semiclassical and experimental values encourages the study of collisions with other ions.

\section{CONCLUSIONS}

In this work we have evaluated the electron emission spectra in collisions of protons with water molecules. We have obtained very good agreement of the two nonperturbative methods applied with experimental data; this supports the relevance of our calculations, particularly those of the CTMC total cross sections for electron production. Our calculations provide a theoretical counterpart to the experiments that, to a certain extent, have not been reproduced theoretically. The good agreement observed indicates that the ionization mechanism in ion-molecule collisions is accurately described with classical mechanics, as previously found in ion-atom collisions [44-47].

Evaluation of singly and doubly differential cross sections in collisions of highly charged projectiles $\left(\mathrm{He}^{2+}, \mathrm{C}^{6+}, \mathrm{O}^{8+}\right)$ with water molecules and other targets of interest in ion beam cancer therapy is in progress.

\section{ACKNOWLEDGMENTS}

This work has been partially supported by Projects No. ENE2007-62934 and No. ENE2011-28200 (Secretaría de Estado de I+D+i, Spain). We thank Professor L. H. Toburen for providing us with the numerical values of his measurements of singly differential cross sections. The Centro de Computación Científica of the Universidad Autónoma de Madrid is acknowledged for computational hosting support.
[1] D. Schardt, T. Elsässer, and D. Schulz-Ertner, Rev. Mod. Phys. 82, 383 (2010).

[2] N. J. Mason, in Radiation Damage in Biomolecular Systems, Proceedings of the 5th International Conference (RADAM 2008), edited by K. Tokési and B. Sulik, AIP Conf. Proc. No. 1080 (AIP, New York, 2008), p. 3.

[3] P. D. Fainstein, G. H. Olivera, and R. D. Rivarola, Nucl. Instrum. Methods B 107, 19 (1996).

[4] B. Gervais, M. Beuve, G. H. Olivera, and M. E. Galassi, Radiat. Phys. Chem. 75, 493 (2006).

[5] O. Boudrioua, C. Champion, C. DalCappello, and Y. V. Popov, Phys. Rev. A 75, 022720 (2007).

[6] C. Champion, O. Boudrioua, C. DalCappello, Y. Sato, and D. Ohsawa, Phys. Rev. A 75, 032724 (2007).

[7] C. DalCappello, C. Champion, O. Boudriuoa, H. Lekadir, Y. Sato, and D. Ohsawa, Nucl. Instrum. Methods B. 267, 781 (2009).

[8] A. Dubois, S. Carniato, P. D. Fainstein, and J. P. Hansen, Phys. Rev. A 84, 012708 (2011).

[9] L. F. Errea, C. Illescas, L. Méndez, B. Pons, I. Rabadán, and A. Riera, Phys. Rev. A 76, 040701 (2007).

[10] C. Illescas, L. F. Errea, L. Méndez, B. Pons, I. Rabadán, and A. Riera, Phys. Rev. A 83, 052704 (2011).

[11] L. F. Errea, C. Illescas, P. Gabás, L. Méndez, I. Rabadán, A. Riera, and B. Pons, in Fast Ion-atom And Ion-molecule Collisions, edited by D. Belkić (World Scientific, Singapore, 2012), pp. 231-266.

[12] M. Murakami, T. Kirchner, M. Horbatsch, and H. J. Lüdde, Phys. Rev. A 85, 052704 (2012).

[13] M. Murakami, T. Kirchner, M. Horbatsch, and H. J. Lüdde, Phys. Rev. A 85, 052713 (2012).

[14] M. Zapukhlyak, T. Kirchner, H. J. Ldde, S. Knoop, R. Morgenstern, and R. Hoekstra, J. Phys. B 38, 2353 (2005).

[15] M. E. Rudd, T. V. Goffe, R. D. DuBois, and L. H. Toburen, Phys. Rev. A 31, 492 (1985).

[16] M. A. Bolorizadeh and M. E. Rudd, Phys. Rev. A 33, 888 (1986).

[17] F. Gobet, S. Eden, B. Coupier, J. Tabet, B. Farizon, M. Farizon, M. J. Gaillard, M. Carré, S. Ouaskit, T. D. Märk et al., Phys. Rev. A 70, 062716 (2004).
[18] H. Luna, A. L. F. de Barros, J. A. Wyer, S. W. J. Scully, J. Lecointre, P. M. Y. Garcia, G. M. Sigaud, A. C. F. Santos, V. Senthil, M. B. Shah et al., Phys. Rev. A 75, 042711 (2007).

[19] L. H. Toburen and W. E. Wilson, J. Chem. Phys. 66, 5202 (1977).

[20] M. Krämer and G. Kraft, Radiat. Environ. Biophys. 33, 91 (1994).

[21] M. Krämer, http://bio.gsi.de/DOCS/trax.html.

[22] D. Emfietzouglou, G. Papamichael, and M. Moscovitch, J. Phys. D: Appl. Phys. 33, 932 (2000).

[23] S. Uehara and H. Nikjoo, J. Phys. Chem. B 106, 11051 (2002).

[24] W. Friedland, H. Paretzke, F. Ballarini, A. Ottolenghi, G. Kreth, and C. Cremer, Radiat. Environ. Biophys. 47, 49 (2008).

[25] L. A. Wehrman, A. L. Ford, and J. F. Reading, J. Phys. B 29, 5831 (1996).

[26] P. M. M. Gabás, L. F. Errea, L. Méndez, and I. Rabadán, Phys. Rev. A 85, 012702 (2012).

[27] B. H. Bransden and M. H. C. McDowell, Charge Exchange and the Theory of Ion-Atom Collisions (Clarendon, Oxford, 1992).

[28] T. Dunning, Jr., J. Chem. Phys. 90, 1007 (1989).

[29] R. A. Kendall, J. Thom H. Dunning, and R. J. Harrison, J. Chem. Phys. 96, 6796 (1992),

[30] See Supplemental Material at http://link.aps.org/supplemental/ 10.1103/PhysRevA.87.032709 for the basis set for the hydrogen projectile and the set of extra diffuse functions on oxygen and hydrogen water molecule atoms.

[31] L. F. Errea, L. Méndez, B. Pons, A. Riera, and I. Sevila, Phys. Rev. A 67, 022716 (2003).

[32] C. Illescas and A. Riera, Phys. Rev. Lett. 80, 3029 (1998).

[33] L. F. Errea, J. D. Gorfinkiel, A. Macías, L. Méndez, and A. Riera, J. Phys. B 30, 3855 (1997).

[34] C. Illescas and A. Riera, J. Phys. B 31, 2777 (1998).

[35] F. Guzmán, L. F. Errea, C. Illescas, L. Méndez, and B. Pons, J. Phys. B 43, 144007 (2010).

[36] H. Luna and E. C. Montenegro, Phys. Rev. Lett. 94, 043201 (2005).

[37] K. H. Tan, C. E. Brion, P. E. Van der Leeuw, and M. J. Van der Wiel, Chem. Phys. Lett. 29, 299 (1978).

[38] U. Werner, K. Beckord, J. Becker, and H. O. Lutz, Phys. Rev. Lett. 74, 1962 (1995). 
[39] F. Gobet, B. Farizon, M. Farizon, M. J. Gaillard, M. Carré, M. Lezius, P. Scheier, and T. D. Märk, Phys. Rev. Lett. 86, 3751 (2001).

[40] T. Kirchner, L. Gulyás, H. J. Lüdde, E. Engel, and R. M. Dreizler, Phys. Rev. A 58, 2063 (1998).

[41] H. J. Lüdde, T. Spranger, M. Horbatsch, and T. Kirchner, Phys. Rev. A 80, 060702 (2009).

[42] B. Boudaïffa, P. Cloutier, D. Hunting, M. A. Huels, and L. Sanche, Science 287, 164 (2000).
[43] L. H. Toburen (private communication).

[44] R. Dörner, H. Khemliche, M. H. Prior, C. L. Cocke, J. A. Gary, R. E. Olson, V. Mergel, J. Ullrich, and H. Schmidt-Böcking, Phys. Rev. Lett. 77, 4520 (1996).

[45] E. Y. Sidky, C. Illescas, and C. D. Lin, Phys. Rev. Lett. 85, 1634 (2000).

[46] C. Illescas, B. Pons, and A. Riera, Phys. Rev. A 63, 062722 (2001).

[47] M. Chassid and M. Horbatsch, Phys. Rev. A 66, 012714 (2002). 